\title{
Magnitudes absolues des étoiles standards MK des types G à M à partir des parallaxes Hipparcos
}

\section{The absolute magnitudes of the G to $M$ type MK standards from the Hipparcos parallaxes}

\author{
N. Ginestet ${ }^{1}$, J.M. Carquillat ${ }^{1}$ et C. Jaschek $^{2}$ \\ 1 Observatoire Midi-Pyrénées, Laboratoire d'Astrophysique (UMR 5572), 14 avenue Edouard Belin, 31400 Toulouse, France \\ 2 Observatoire de Strasbourg, URA 1280 (CNRS), 11 rue de l'Université, 67000 Strasbourg, France
}

Reçu le 20 juillet ; accepté le 10 novembre, 1999

\begin{abstract}
We analyse a sample of about 500 MK standards of cool spectral types ( $\mathrm{G}$ to $\mathrm{M}$ ) for to compare the visual absolute magnitudes obtained from both Hipparcos data and Schmidt-Kaler calibrations. Our purpose is to validate our spectroscopic work (Ginestet et al. 1997, 1999) on stars with composite spectra with the help of Hipparcos data.

Contrary to what is claimed in other papers, the absolute magnitude domain devoted to the giant stars does not overlap the domain of dwarfs. We find that the discrepancies between absolute magnitudes from Hipparcos data and absolute magnitudes deduced from Schmidt-Kaler calibrations increase with the relative error $\sigma(\pi) / \pi$ on the parallaxes. So, for $\sigma(\pi) / \pi \leq 0.05$ only $3 \%$ of the stars present a discrepancy of one luminosity class, while this percentage reaches $54 \%$ for $0.25<\sigma(\pi) / \pi \leq 0.50$.
\end{abstract}

Curiously, the luminosity of the giants seems to increase with the distance of the stars, whereas the supergiants of the sample appear underluminous at least for $d<600 \mathrm{pc}$ !

We point out a list of $14 \mathrm{MK}$ standards whose luminosity classes may be erroneous and need a new spectral classification, in the near infrared. The case of compositespectrum binaries is also discussed. Most of these are too distant for accurate parallaxes even with Hipparcos: only sixteen stars have $\sigma(\pi) / \pi \leq 0.10$; for these, we give new spectral classifications in agreement with both our classifications in the near infrared of the cool components and Hipparcos data.

Finally, for stars having high-precision parallaxes $(\sigma(\pi) / \pi \leq 5 \%)$ there is no serious problem for SchmidtKaler calibrations whith respect to Hipparcos data. The data corresponding to parallaxes of lower precisions should be used with caution and only for statistical analyses.
Key words: stars: fundamental parameters - stars: distances

\section{Introduction}

Les données Hipparcos présentaient un double intérêt pour nos travaux sur les étoiles à spectre composite, systèmes binaires constitués d'une étoile naine chaude de type $\mathrm{B}$ ou A et d'une géante ou supergéante froide de type $\mathrm{G}, \mathrm{K}$ ou $\mathrm{M}$ :

- tester nos classifications, effectuées dans le proche infrarouge (Ginestet et al. 1997, 1999), des composantes froides d'un petit échantillon de ces binaires pour lesquelles les classifications des composantes chaudes étaient bien connues ;

- et, si ce premier test était concluant, déduire des données Hipparcos les classifications des composantes chaudes d'un échantillon plus large d'étoiles à spectre composite. Notre première tentative d'utiliser en ce sens les données d'Hipparcos (Carquillat et al. 1997) s'est révélée décevante, ce qui nous a conduits à examiner pour les étoiles standards MK de types G, K, M l'accord ou le désaccord existant entre les données Hipparcos et les données issues des calibrations $M_{v}$ /Type MK de Schmidt-Kaler (1982).

De récents travaux ont déjà été effectués dans ce sens, dont deux se rapportent aux étoiles chaudes (Jaschek \& Gómez 1998; Paunzen 1999), les échantillons étant constitués uniquement de standards MK ; un autre travail (Gómez et al. 1997) porte sur un échantillon beaucoup plus important de quelque 22000 étoiles observées par Hipparcos, de types $\mathrm{B}$ à $\mathrm{K}$ et possédant une classification MK. Pour les deux premières études, l'un des critères de sélection des échantillons est la limite admise pour l'erreur $\sigma\left(M_{v}\right)$ sur les magnitudes absolues déduites des parallaxes trigonométriques, quantité directement liée à 
l'erreur relative $\sigma(\pi) / \pi$ sur la parallaxe : ainsi Jaschek \& Gómez ont-ils adopté comme condition $\sigma\left(M_{v}\right) \leq 0,3$ mag, soit $\sigma(\pi) / \pi \leq 0,14$ et Paunzen une valeur légèrement supérieure $\sigma(\pi) / \pi \leq 0,18$, soit $\sigma\left(M_{v}\right) \leq 0,4$ magnitude ; par contre Gómez et al. utilisent une méthode statistique mettant en jeu non seulement les parallaxes trigonométriques elles-mêmes mais également des données cinématiques (mouvements propres et vitesses radiales), cette méthode permettant, selon eux, une amélioration de l'estimation des magnitudes absolues qui autorise l'extension de l'échantillon à toutes les parallaxes disponibles, y compris celles de faibles précisions.

Nous pouvons résumer en ces termes les principales conclusions qui ressortent de ces études :

1) la dispersion en magnitudes, vis-à-vis des séquences "traditionnelles" du diagramme HR, des étoiles observées par Hipparcos est beaucoup plus importante qu'on ne le pensait auparavant, en particulier pour la séquence principale ;

2) on constate un large empiétement entre les séquences des naines et des géantes, ce qui remet en cause l'existence de la classe IV ;

3) l'ampleur de cette dispersion des magnitudes remet fortement en question la fiabilité de l'utilisation des calibrations (par exemple celles de Schmidt-Kaler) en $M_{v}$ des différentes classes de luminosité quant à l'estimation de la magnitude absolue d'une étoile à partir de son type spectral MK.

C'est donc l'un des aspects potentiels majeurs du système MK ayant contribué à assurer jusqu'ici sa pérennité, en l'occurrence son lien avec les paramètres physiques fondamentaux des étoiles, qui se trouve ainsi mis à mal. L'importance de cette question, en plus des motivations exposées plus haut, nous a conduits à effectuer cette nouvelle investigation sur un échantillon, sans équivalent dans les travaux précédents, composé uniquement de standards MK des types froids ( $G$ à $M)$. Nous avons aussi abordé d'une façon différente le problème de la limitation de l'échantillon suivant la valeur de $\sigma(\pi) / \pi$.

\section{L'échantillon}

Toutes les étoiles de notre échantillon proviennent de la liste de standards MK de Garcia (1989) : au total nous avons répertorié 513 étoiles de types $\mathrm{G}, \mathrm{K}, \mathrm{M}$ des classes de luminosité Ia, Ib, II, III, IV, V ayant été observées avec Hipparcos. Les standards de classes de luminosité intermédiaires, telles Ib-II, II-III, etc. ont été éliminées. Signalons deux erreurs, détectées dans la liste de B. Garcia, concernant les étoiles HD 50281 et HD 125454 : - HD 50281, binaire visuelle : HD 50281B = Gliese 250B est une standard M2V (Keenan \& McNeil 1976) de magnitude visuelle 10,05 mais les paramètres indiqués dans la liste (magnitude, indice de couleur) sont ceux de HD 50281A (K3V) qui, elle, n'est pas standard MK ; les

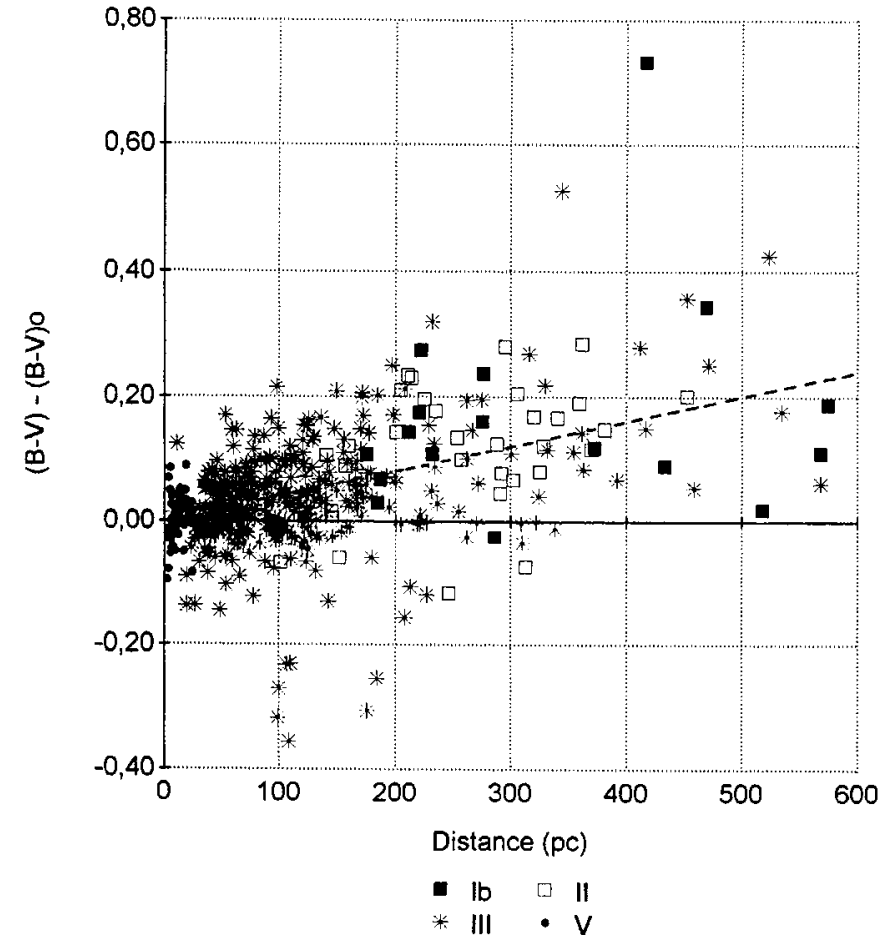

Fig. 1. Évolution de l'excès de couleur $(B-V)-(B-V)_{0}$, pour un échantillon de standards MK, en fonction de la distance ; $(B-V)$, donnée Hipparcos et $(B-V)_{0}$, indice de couleur de Schmidt-Kaler (1982)

données Hipparcos concernent HD 50281A ;

- HD 125454 (Ups Vir) listée M1III est en fait de type G8III (cf. SIMBAD du CDS) et n'est pas standard MK.

Notre échantillon se réduit donc à 511 étoiles dont la répartition en types spectraux et en classes de luminosité est la suivante :

\begin{tabular}{|c|c|c|c|c|}
\hline SP & G & K & M & total \\
\hline Ia & 1 & & 1 & 2 \\
\hline Ib & 14 & 8 & 4 & 26 \\
\hline II & 13 & 24 & 8 & 45 \\
\hline III & 98 & 172 & 88 & 358 \\
\hline IV & 12 & 5 & & 17 \\
\hline V & 33 & 20 & 10 & 63 \\
\hline total & 171 & 229 & 111 & 511 \\
\hline
\end{tabular}

- Excès de couleur, absorption interstellaire

Afin d'évaluer l'incidence du rougissement interstellaire sur notre échantillon, nous avons calculé, pour chaque standard, l'excès de couleur $E_{B-V}=(B-V)-(B-V)_{0}$ où $(B-V)_{0}$ est l'indice de couleur intrinsèque correspondant au type spectral MK d'après les calibrations de SchmidtKaler (1982) et $B-V$ l'indice relevé dans le catalogue Hipparcos (obtenu soit de mesure au sol, soit de mesure avec Tycho). L'évolution de l'excès de couleur en fonction 
de la distance est donné en Fig. 1 : les points présentent une certaine dispersion autour d'une droite de faible pente qui représente, en moyenne, l'augmentation de $E_{B-V}$ avec la distance ; un effet de blanketing paraît exclu car la plupart des étoiles de l'échantillon figurant dans le catalogue de Cayrel et al. (1992) ont des métallicités solaires $(-0,4<[\mathrm{Fe} / \mathrm{H}]<+0,4)$. Ceci nous a donc permis de corriger de l'absorption interstellaire mais l'évaluation de cette correction n'étant plus possible, par manque d'objets, audelà d'une distance d'environ 600 pc, 19 étoiles (dont les deux supergéantes Ia de l'échantillon) seront éliminées en ce qui concerne les magnitudes absolues (Sects. 3.2 et 3.4).

Le cas des étoiles ayant des $E_{B-V}$ fortement négatifs qui pourraient indiquer la présence de compagnons plus chauds, sera étudié au paragraphe 3.3.

\section{- Précision des parallaxes}

L'erreur relative sur la parallaxe $\sigma(\pi) / \pi$ croît, en moyenne, avec la distance, mais également la dispersion des valeurs de ce rapport autour des valeurs moyennes augmente avec la distance des objets (Fig. 2). La répartition des 511 standards MK selon $\sigma(\pi) / \pi$ est la suivante :



Fig. 2. Erreur relative sur les parallaxes Hipparcos $(\sigma(\pi) / \pi)$ en fonction de la distance

\begin{tabular}{|c|c|c|c|c|c|c|c|}
\hline$\sigma(\pi) / \pi$ & $0-0,05$ & $0,06-0,10$ & $0,11-0,15$ & $0,16-0,20$ & $0,21-0,25$ & $0,26-0,30$ & $>0,30$ \\
\hline$N$ & 210 & 130 & 64 & 33 & 20 & 13 & 41 \\
\hline
\end{tabular}

\section{Données Hipparcos-Tycho}

\section{- Binarité}

La binarité fausse l'estimation des magnitudes absolues, mais on peut supposer que ce biais ne dépend pas de la distance et affecte de façon homogène tout l'échantillon. Cependant, pour les objets dont la binarité était connue (binaires visuelles serrées et binaires spectroscopiques) nous avons effectué une correction en utilisant les mêmes critères que Jaschek \& Gómez (1998) ainsi que les informations issues du Bright Star Catalogue (Hoffleit \& Jaschek 1982), du Catalogue Hipparcos (ESA 1997) et du Catalogue de Batten et al. (1989).

Dans le cas des binaires spectroscopiques à un seul spectre (binaires avec orbite connue ou étoiles à vitesse radiale variable) une correction de $+0,2$ mag a été appliquée, ce qui correspond à $\Delta m=1,75$ mag. Aucune correction n'a été faite pour les étoiles à baryum binaires, leurs compagnons étant supposés être des étoiles de très faibles luminosités (naines blanches).

Notons, comme l'indiquent Jaschek \& Gómez (1998), qu'une des difficultés pour corriger l'effet de la binarité tient au fait qu'elle n'est toujours qu'imparfaitement connue pour un échantillon donné.

\subsection{Parallaxes : comparaison avec les données au sol}

Parmi les 511 étoiles de l'échantillon, 357 avaient une parallaxe mesurée au sol reportée dans le General Catalogue of Trigonometric Stellar Paralaxes (van Altena et al. 1995).

La comparaison des parallaxes Hipparcos et des parallaxes sol pour ces étoiles est illustrée aux Figs. 3a et $3 \mathrm{~b}$ où les distances sont limitées respectivement à 500 et 50 parsecs. On constate :

- jusqu'à environ 15 pc un excellent accord entre les deux séries de parallaxes ;

- pour $d>15$ pc la dispersion des points autour de la bissectrice augmente avec la distance, mais cette dispersion présente un caractère dissymétrique : statistiquement, les distances déterminées à l'aide des parallaxes Hipparcos sont plus fortes que celles obtenues avec les parallaxes mesurées au sol. Ce fait était déjà mis en évidence par C. Turon (1998) à partir de deux échantillons : les étoiles du Catalogue of Nearby Stars, et des étoiles naines du Michigan Spectral Survey estimées être à moins de 80 pc du Soleil d'après leurs parallaxes spectroscopiques. 
a)





Fig. 3. Comparaison entre les parallaxes-Hipparcos et les parallaxes-sol ; a) la distance est limitée à 500 pc, b) à 50 pc

\subsection{Magnitudes absolues : confrontation aux calibrations de Schmidt-Kaler}

Dans ce qui suit nous présentons, sous forme de graphiques, une comparaison des magnitudes absolues déduites des parallaxes Hipparcos $\left(M_{\text {Hip }}\right)$ à celles données par les calibrations de Schmidt-Kaler $\left(M_{\mathrm{SK}}\right)$. Ces comparaisons sont faites successivement sur plusieurs échantillons correspondant à différentes limites en $\sigma(\pi) / \pi$.

Afin d'effectuer la confrontation avec le plus de précision possible nous avons procédé, en ce qui concerne les $M_{\mathrm{SK}}$, aux interpolations indiquées par Keenan \& McNeil (1976) pour les standards ayant des classes de luminosité accompagnées des suffixes a, b, ab.

- Diagrammes $M_{H i p} / M_{S K}$

Sur la Fig. 4a nous constatons pour les magnitudes Hipparcos une grande dispersion des points (sauf pour les naines) mais pas de recoupements entre naines et géantes à l'exception d'une seule étoile HD 204613, objet très particulier ; la classe IV se situe normalement entre les classes V et III avec une dispersion plus grande que pour les naines. Notons que HD $204613(\sigma(\pi) / \pi=0,11)$ est une étoile de type G1IIIa: $\mathrm{CH} 1,5$ dont les données photométriques et spectroscopiques sont en nette opposition (Bond 1970a, 1970b) : la photométrie correspondrait à celle d'une naine et le spectre indiquerait une classe III. Deux autres étoiles géantes sortent aussi nettement du groupe mais, cette fois, en direction de la classe II ; ce sont HD 81817 et HD 176670 :

- HD 81817, K3IIIa, $B-V=1,488(\sigma(\pi) / \pi=0,18)$ est située à environ $330 \mathrm{pc}$ et l'indice de couleur paraît fort pour une classe III, même en tenant compte du rougissement ;

- HD 176670, K2,5III Ba0,5, $B-V=1,465$ $(\sigma(\pi) / \pi=1,26), 422 \mathrm{pc}$ : comme pour HD 81817 l'indice $B-V$ semble élevé, de plus plusieurs classifications K3II figurent dans les données du CDS.

Ces deux étoiles devraient être reclassées dans l'infrarouge.

En contraignant l'échantillon par différentes limites de $\sigma(\pi) / \pi$ nous constatons que plus cette quantité est petite plus les données Hipparcos sont en bon accord avec celles de Schmidt-Kaler (Figs. 4b, c, d), mais pour les plus faibles valeurs de $\sigma(\pi) / \pi$ il ne reste évidemment que les classes de luminosité V, IV et III, avec de moins en moins d'objets de la classe des géantes.

- Diagrammes $\left(M_{\mathrm{Hip}}-M_{\mathrm{SK}}\right) /(\sigma(\pi) / \pi)$

Nous étudions ici le comportement de la différence $D=$ $M_{\mathrm{Hip}}-M_{\mathrm{SK}}$ en fonction de l'erreur relative sur la parallaxe (Fig. 5a). Nous constatons que :

- tant que l'erreur relative sur les parallaxes ne dépasse pas $5 \%(\sigma(\pi) / \pi \leq 0,05)$, soit $d \cong 75$ pc (Fig. 5b), les points sont distribués de part et d'autre de la droite $D=0$, avec une plus forte concentration à son voisinage. 
a)

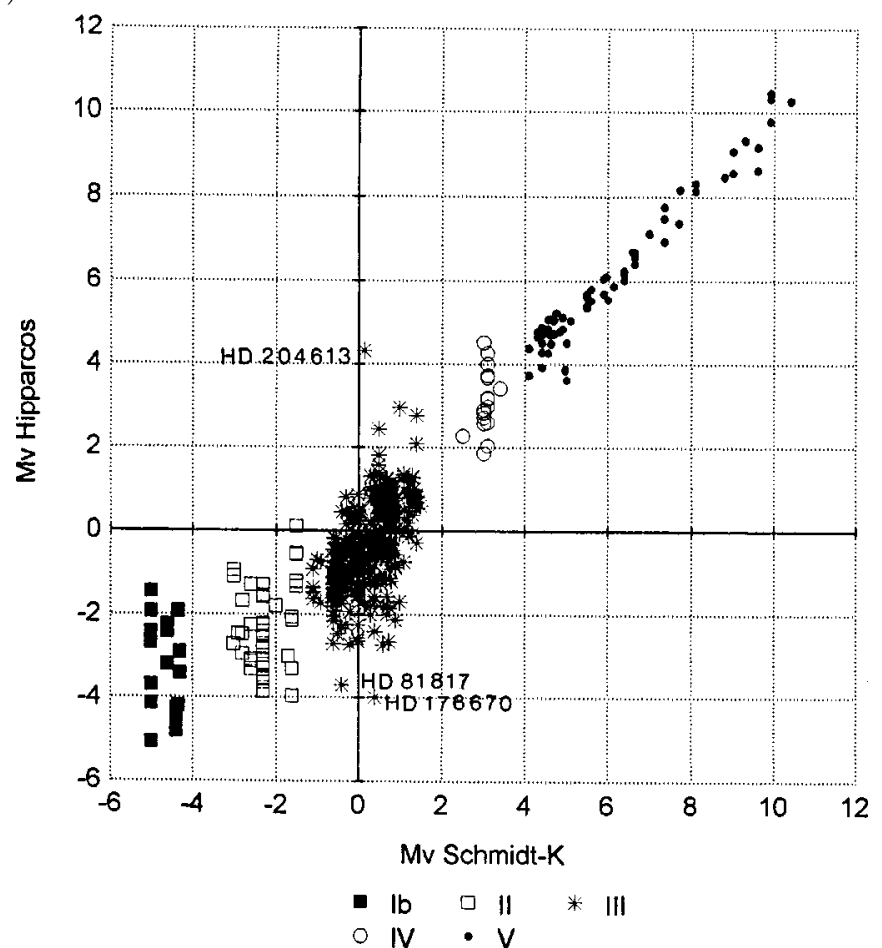

c)



b)

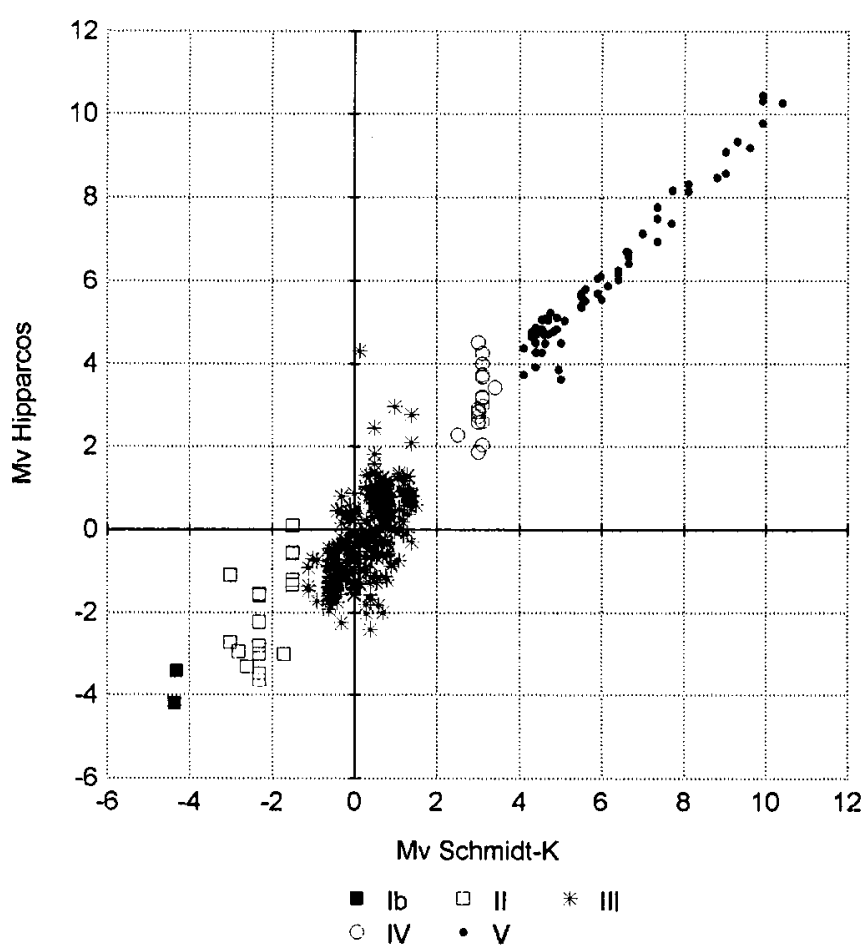

d)

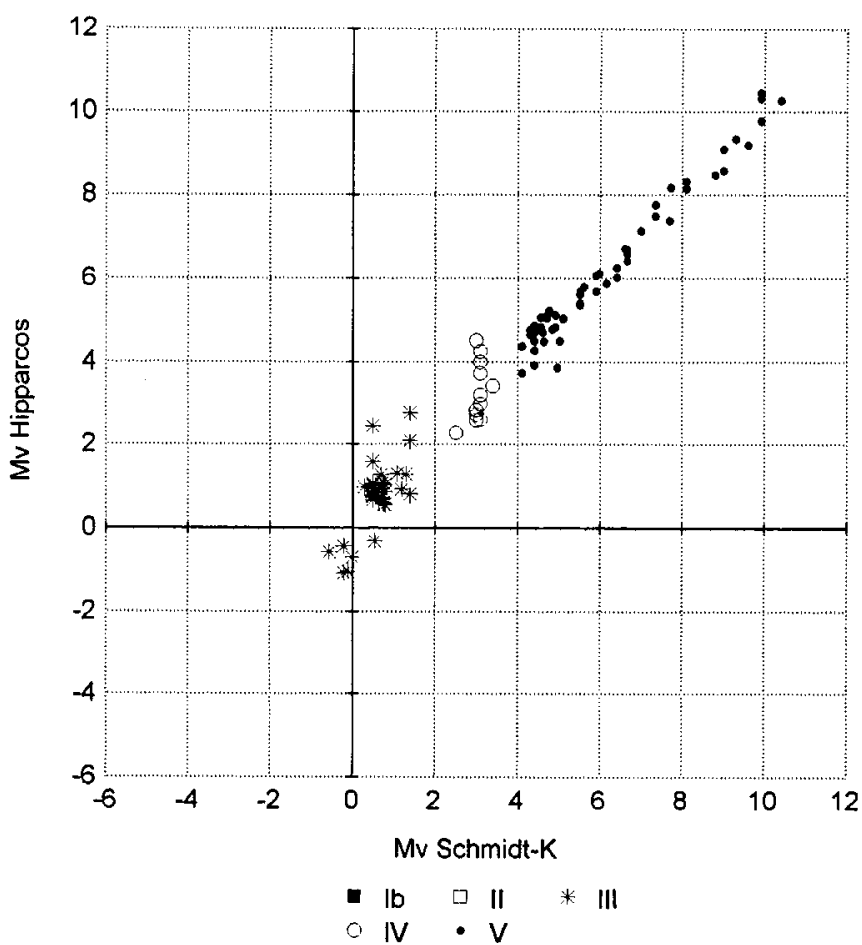

Fig. 4. Confrontation entre les magnitudes absolues Hipparcos et celles de Schmidt-Kaler ; a) comporte toutes les étoiles de l'échantillon, b, c, d) correspondent à des limitations en $\sigma(\pi) / \pi$ respectivement de 0,$15 ; 0,05$ et 0,02 . A la Fig. 4a, 3 étoiles géantes sortent nettement de ce groupe : voir Sect. 3.2 
a)

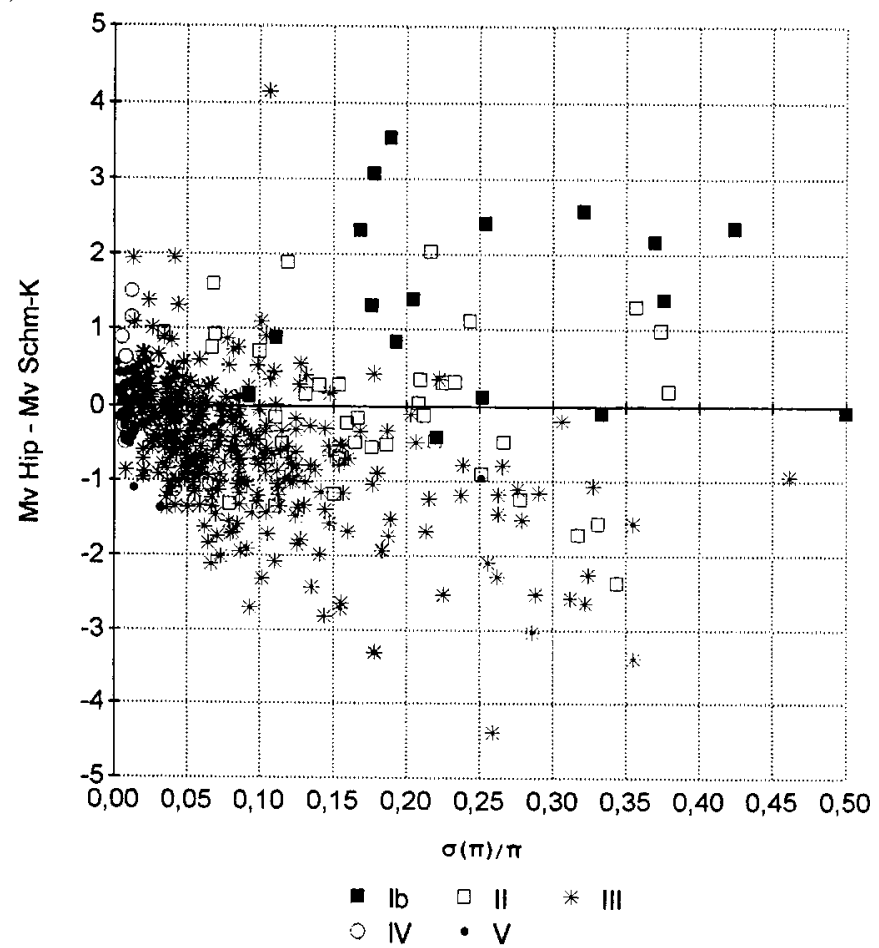

c)



b)



d)

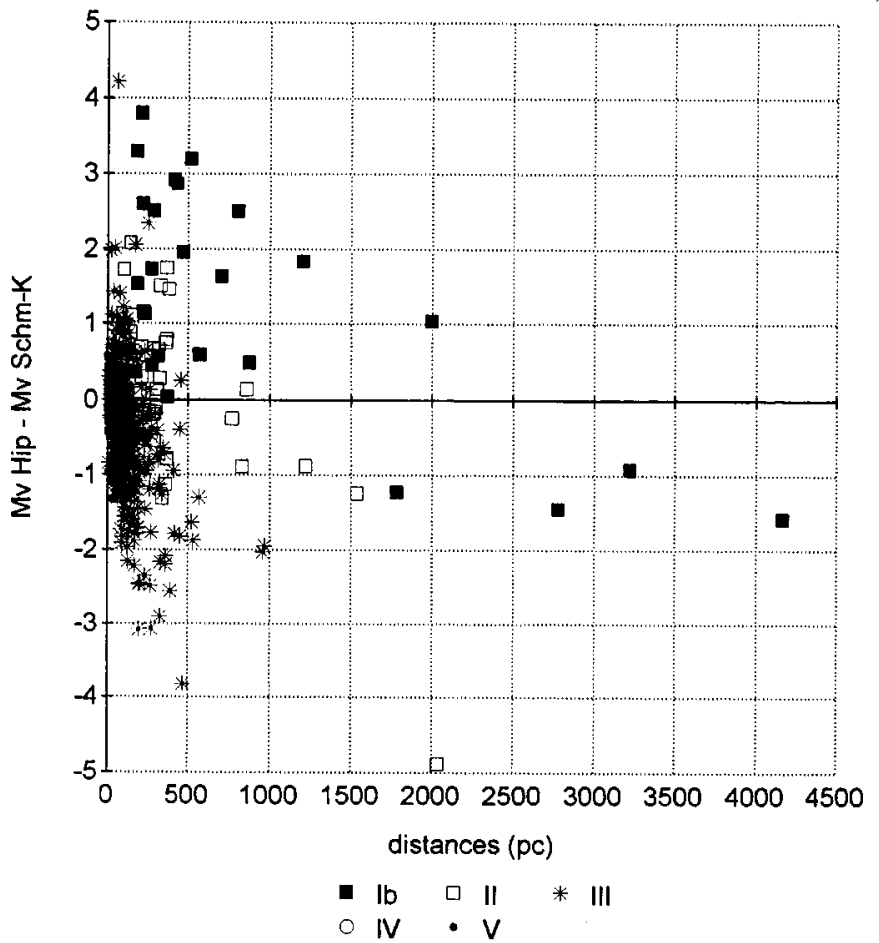

Fig. 5. Comportement de la différence entre magnitudes absolues Hipparcos et Schmidt-Kaler en fonction de l'erreur relative sur la parallaxe $(\mathbf{a}) \sigma(\pi) / \pi<0,50 ; \mathbf{b}) \sigma(\pi) / \pi<0,05)$ et de la distance (c) distance limitée à 600 pc ; d) ensemble de l'échantillon) 
a)

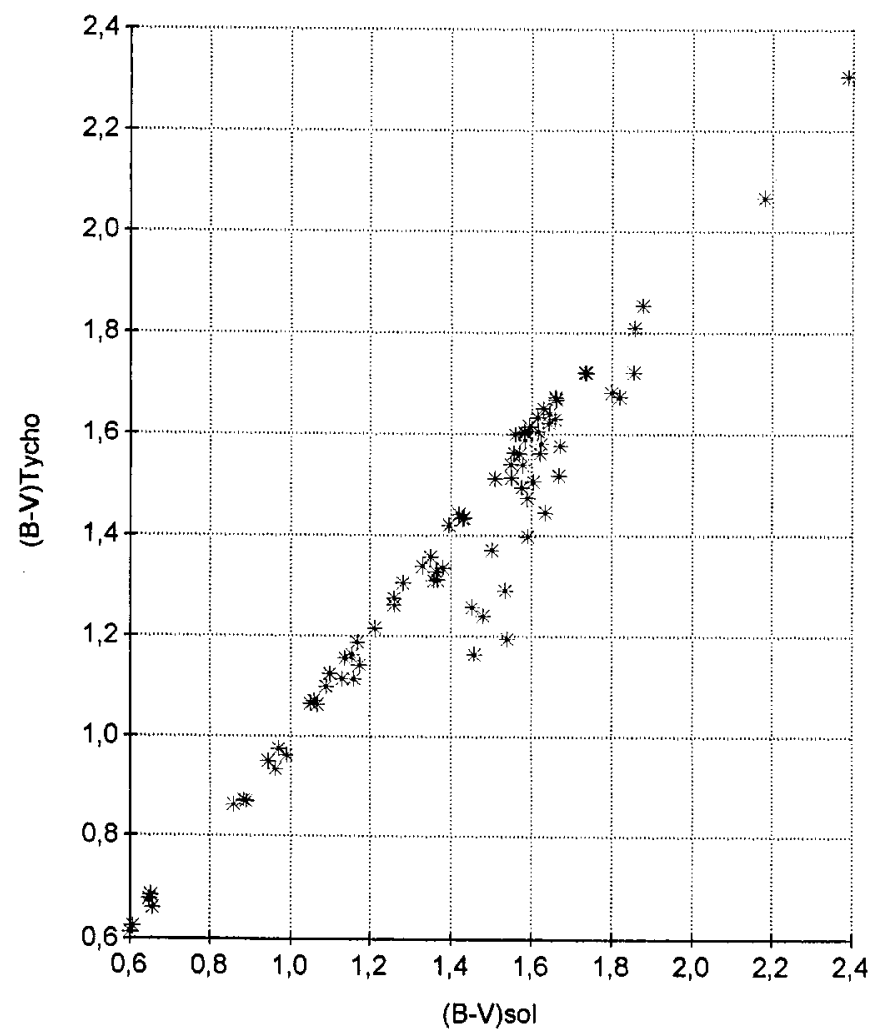

$\mathrm{GO} .0 \mathrm{KO} 0.1 \mathrm{MO} 0.2$



Fig. 6. a) comparaison entre les indices de couleur $(B-V)$ Tycho et les indices $(B-V)$ sol, b) différence entre les indices-Tycho et les indices-sol en fonction des types spectraux

Ceci est vrai pour les naines comme pour les géantes, sauf quelques cas isolés sur lesquels nous reviendrons. À ces faibles distances, nous ne trouvons pas de supergéante dans l'échantillon, et une seule géante brillante (classe IIb).

- pour $\sigma(\pi) / \pi>0,05$ les géantes ont un comportement pour le moins surprenant : les points représentatifs de ces étoiles "descendent" en dessous de la droite $D=0$, "descente" qui semble même s'amorcer vers $\sigma(\pi) / \pi=0,035$. En d'autres termes, les géantes seraient plus brillantes quand elles sont plus éloignées ! et comme les distances concernées sont relativement faibles, à partir d'environ 75 pc, ce comportement peut difficilement être attribué à un simple biais observationnel. Cette "dérive" en fonction de l'erreur relative sur la parallaxe semble aller dans le même sens pour les géantes brillantes. Les supergéantes de l'échantillon sont, quant à elles, trop peu nombreuses et trop dispersées sur le diagramme pour une analyse du comportement du groupe en fonction de $\sigma(\pi) / \pi$ : on remarque simplement que, contrairement aux géantes, les supergéantes présenteraient, en moyenne et pour l'intervalle considéré, un "déficit" de luminosité par rapport aux valeurs de Schmidt-Kaler.

Nous illustrons également cette différence $D$ des magnitudes absolues en fonction de la distance aux Figs. 5c et 5 d. La Fig. 5 c est réalisée avec des $M_{\text {Hip }}$ corrigées de la binarité et de l'absorption interstellaire (comme 5a et 5b) l'échantillon étant limité à 600 pc. Par contre la Fig. 5d est constituée avec l'échantillon complet et donc les $M_{\text {Hip }}$ n'ont été corrigées que de la binarité car les distances allant jusqu'à près de 4500 pc le nombre d'étoiles compris entre 600 et 4500 pc est trop faible pour évaluer de façon correcte l'absorption. Cette dernière figure montre nettement la tendance de la différence $D$ à décroître avec la distance et ceci pour toutes les luminosités, tendance qui serait encore renforcée si on introduisait une correction pour l'absorption interstellaire!

\subsection{Indices de couleur $B-V$}

Sur les 511 étoiles de l'échantillon 92 seulement ont des mesures de $B-V$ avec Tycho et, parmi ces 92 objets, 12 n'ont pas de mesure de $B-V$ au sol. La comparaison des indices-sol et des indices-Tycho est donc faite à partir de 80 étoiles et est illustrée à la Fig. 6a.

Pour un certain nombre d'étoiles les indices-Tycho sont sensiblement inférieurs aux indices-sol : il s'agit surtout de géantes rouges plus tardives que M4, variables pulsantes de type Mira pour la plupart, mais il y a également quelques supergéantes Ib. La Fig. $6 \mathrm{~b}$ présente les différences d'indices $(B-V)$ Tycho $-(B-V)$ sol en 
a)

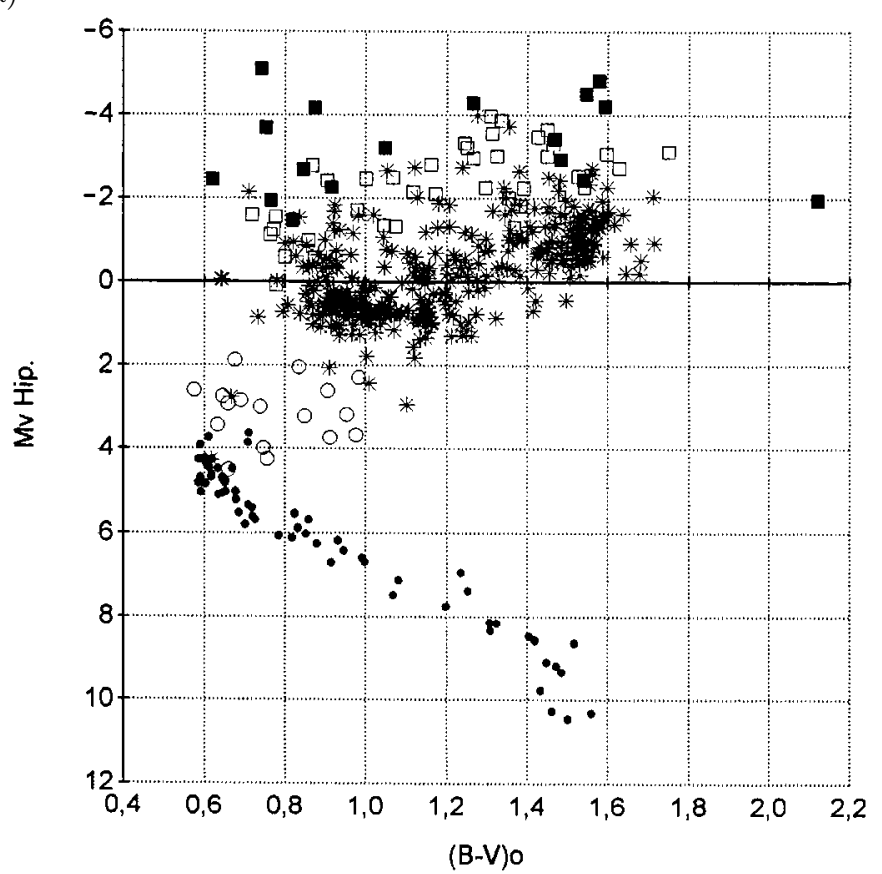

- Ib $\square$ II $\quad$ III

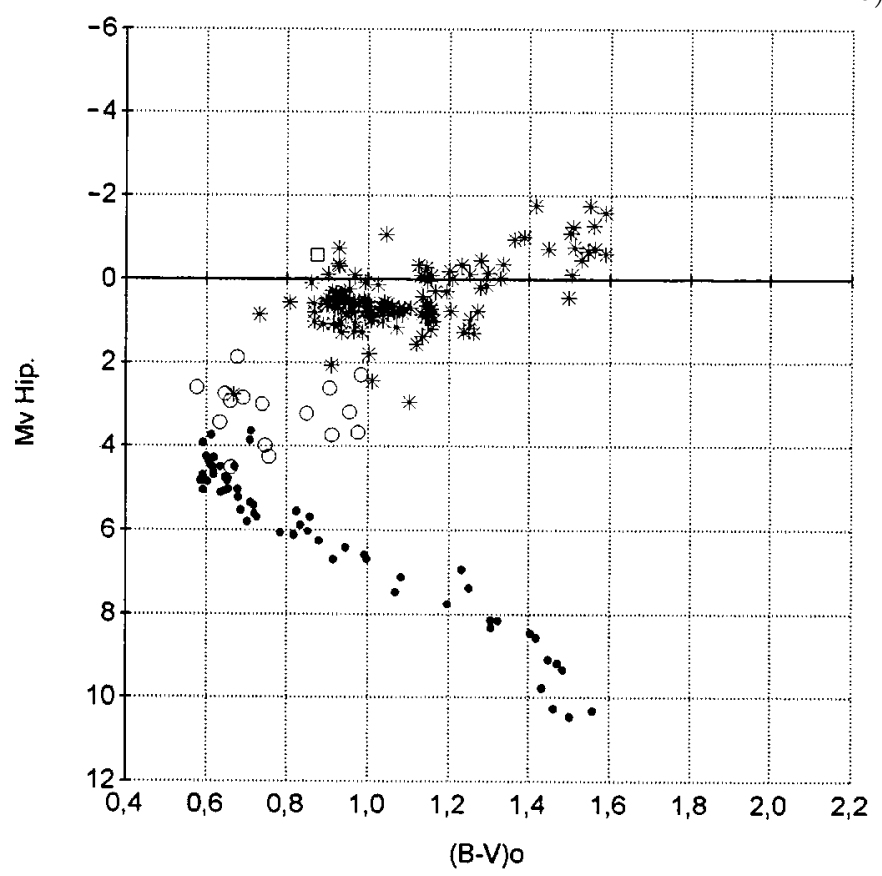

- Ib 0 II $*$ III

b)

Fig. 7. Diagrammes HR $\left.\left(M_{v} \text { Hip./( } B-V\right)_{0}\right)$ : a) avec l'échantillon complet, b) avec $\sigma(\pi) / \pi \leq 0,05$

a)



b)

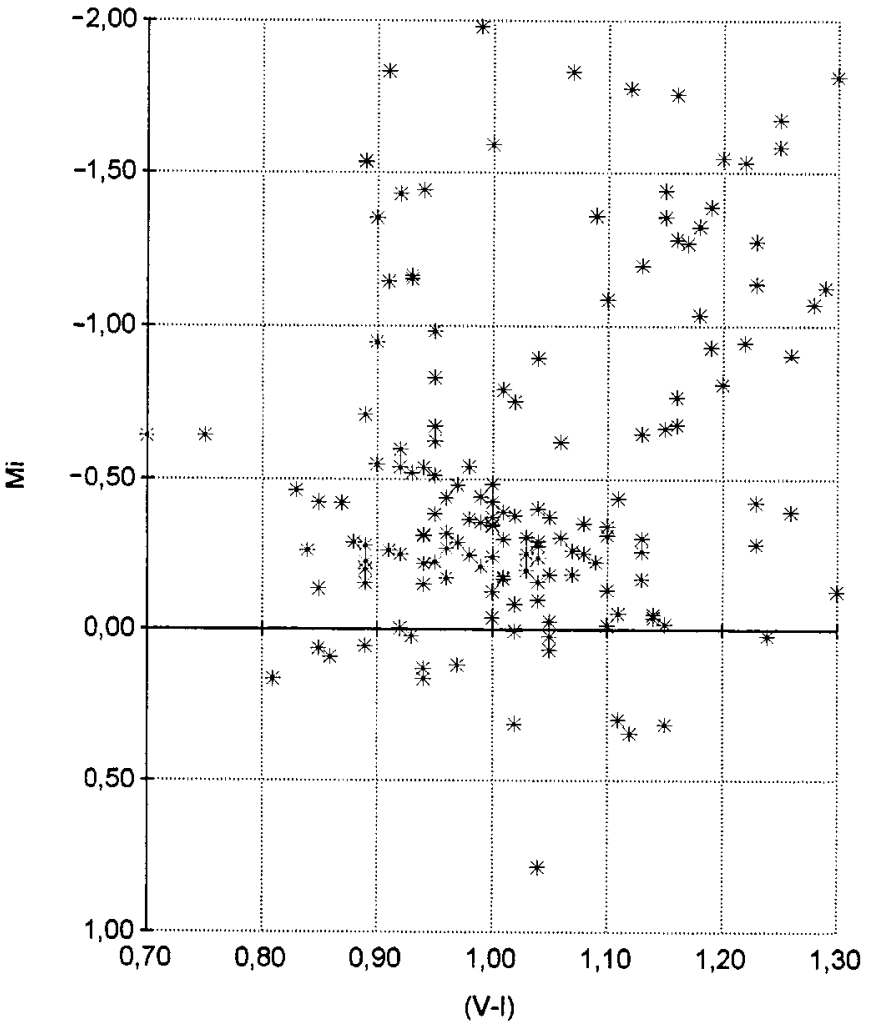

Fig. 8. Répartition des Standards MK géantes rouges en fonction de l'indice de couleur $V-I$ pour comparaison avec celle de Girardi et al. (1998) : voir texte Sect. 3.4 ; a) $\sigma(\pi) / \pi<0,05$ et b) $\sigma(\pi) / \pi<0,10$ 
fonction des types spectraux : ci-dessous nous identifions les objets pour lesquels ces différences sont plus négatives que $-0,075$.

Ce sont : HD 94613 (M3+Ib), 94705 (M5,5III), 108849 (M7-III), 111499 (M4,5II), 122250 (M6,5III), 123657 (M4,5III), 148783 (M6-III), 151732 (M4,5III), 160371 (K2Ib H1), 172380 (M4,5-M5+II), 175588 (M4II), 175865 (M5IIIv), 194258 (M4,5-M5III), 196610 (M6III), 196819 (K2,5IIb) 202380 (M2-Ib), 204585 (M4,5IIIa), 221861 (G9Ib), donc 4 supergéantes, 4 géantes brillantes et 10 géantes (ces dernières étant toutes plus tardives que M4).

Pour les géantes $\mathrm{M}$ tardives les $B-V$ mesurés au sol, souvent plusieurs valeurs proches pour une même étoile, proviennent de SIMBAD et sont, quant à eux, en bon accord avec les classifications spectrales.

On pourrait attribuer les écarts constatés entre $(B-$ $V)$ sol et $(B-V)$ Tycho à des problèmes de conversion des magnitudes Tycho en magnitudes $B, V$ du système de Johnson, mais d'après les indications du catalogue Hipparcos (Vol. 1), cette conversion nécessite une bonne connaissance de la classification spectrale, ce qui est le cas puisque les objets concernés sont des standards MK!

\subsection{Diagramme $H R$}

Les Figs. 7a,b montrent des diagrammes HR ( $M_{\text {Hip }}$ $\left./(B-V)_{0}\right)$ effectués respectivement pour l'échantillon complet et pour un échantillon limité à $\sigma(\pi) / \pi \leq 0,05$, où $(B-V)_{0}$ est l'indice de couleur Hipparcos corrigé du rougissement (cf. Sect. 2). On constate là aussi que la séquence des géantes s'affine lorsque l'on diminue la limite pour $\sigma(\pi) / \pi$, et, encore une fois, on n'observe pas de chevauchement entre la zone des naines et celle des géantes ; le chevauchement entre les géantes et les supergéantes reste très marginal.

Confrontation avec les travaux de Girardi et al. (1998) Girardi et al. (1998) ont étudié la structure fine du groupe des géantes rouges à partir d'un échantillon de 2546 étoiles sélectionnées dans le Catalogue Hipparcos (ESA 1997) : ils comparent les diagrammes $\operatorname{HR}\left(M_{i} / V-I\right)$ obtenus avec des limitations de $\sigma(\pi) / \pi$ de 0,05 et 0,1 à des diagrammes similaires obtenus par une simulation. La théorie utilisée met en jeu plusieurs catégories de géantes rouges de masses différentes. Pour résumer, cette théorie prévoit un parcours évolutif différent suivant que la masse de l'étoile est supérieure ou inférieure à une valeur voisine de $2 M_{\odot}$, avec en corollaire des différences de luminosités qui vont se traduire par une dispersion caractéristique des points sur les diagrammes.

Avec notre échantillon, réduit aux seules étoiles géantes (classe III) et en adoptant les mêmes limitations en $\sigma(\pi) / \pi$, nous trouvons une structure (Figs. 8a, b) qui peut être rapprochée de celle trouvée, avec leur échantillon, par Girardi et al. Comme ces derniers concluent à un bon accord entre la structure observée et celle prédite par la théorie, si le processus impliqué était confirmé il pourrait peut-être expliquer, en partie, la dispersion des magnitudes absolues des géantes, et la calibration de Schmidt-Kaler s'appliquerait essentiellement aux étoiles du coeur compact de la structure.

\section{Discussion}

\subsection{Standards $M K$}

La confrontation précédente des magnitudes absolues $M_{v}$ obtenues à partir des données Hipparcos avec celles de Schmidt-Kaler (Sect. 3.2 et Fig. 5a) permet d'identifier certaines standards MK dont les classifications spectrales pourraient être erronées et pour quelques autres de préciser leurs classifications. Elle nous permet également d'établir des statistiques concernant la fiabilité des données pour les standards MK froides et donc de définir une liste de ces étoiles pour lesquelles les données sont "sûres", c'est-à-dire des étoiles pour lesquelles il y a accord entre Schmidt-Kaler et Hipparcos.

On peut considérer qu'il y a accord lorsque la valeur absolue de la différence $M_{v}$ Hip $-M_{v}$ sK est inférieure ou égale à 0,8 magnitude ; cette valeur moyenne correspond à une subdivision en classe de luminosité (ex. : K0III... $M_{v}=0,7$; IIIa... - 0,1; etc.) au voisinage du type K0III (Keenan \& McNeil 1976).

Sur 486 étoiles concernées $(\sigma(\pi) / \pi \leq 0,50) 344$ objets ont des données cohérentes et il est intéressant de considérer la répartition de l'ensemble de l'échantillon selon le critère $\sigma(\pi) / \pi$ :

- $\sigma(\pi) / \pi \leq 0,05$.

Pour cette zone l'accord entre les données Hipparcos et celles de Schmidt-Kaler peut être qualifié de très bon : sur un total de 209 étoiles seulement 6 objets (soit $3 \%$ ) auraient un désaccord d'une classe de luminosité et 17 (8\%) une demi-classe de luminosité.

- $0,06 \leq \sigma(\pi) / \pi \leq 0,10$.

Cette seconde zone paraît pouvoir aussi être utilisée pour faire quelques contrôles des standards MK. Sur 128 étoiles 8 objets $(6,3 \%)$ sont en désaccord d'une classe de luminosité et $29(22,7 \%)$ d'une demi-classe.

Nous donnons en Tableau 1 une liste des 14 étoiles, concernant ces deux zones, pour lesquelles les classifications pourraient être erronées d'une classe de luminosité et pour lesquelles il serait donc souhaitable d'obtenir des classifications dans le proche infrarouge.

Il est à noter, d'ailleurs, que HD 27022, standard G5II, $(\sigma(\pi) / \pi=0,07)$ avec les données Hipparcos apparaît être une étoile de classe III et non II, ce que notre étude dans l'infrarouge (Ginestet et al. 1994) avait déjà mis en évidence. 
Tableau 1. Standards MK avec $\sigma(\pi) / \pi \leq 0,10$ et ayant un désaccord d'une classe de luminosité entre données Hipparcos et calibrations Schmidt-Kaler. Les valeurs des magnitudes déduites des données Hipparcos $\left(M_{v} H_{i p} .^{*}\right)$ ont été corrigées du rougissement et de la binarité (voir Sect. 2)

\begin{tabular}{|c|c|c|c|c|c|c|c|c|c|c|c|}
\hline HD & $\mathrm{N}^{\circ} \mathrm{HIPP}$. & $\boldsymbol{V}$ & $B-V$ & $\underset{\text { mas }}{\pi}$ & $\begin{array}{l}\sigma(\pi) \\
\text { mas }\end{array}$ & $\sigma(\pi) / \pi$ & $\begin{array}{c}\text { distance } \\
p c\end{array}$ & $M_{V}$ Hip. * & SPS SUd MK & Sp proposé & Notes (CDS) \\
\hline 1522 & 1562 & 3,56 & 1,214 & 11,26 & 0,73 & 0,06 & 89 & $-1,29$ & $K 1,5 I I I / K I I I l b$ & $\mathrm{~K} 1,5 \mathrm{IIb}$ & variable star \\
\hline 27022 & 20266 & 5,26 & 0,820 & 9,80 & 0,67 & 0,07 & 102 & 0,09 & GS IIb & G5IIIa & \\
\hline 40409 & 27890 & 4,65 & 1,022 & 36,67 & 0,48 & 0,01 & 27 & 2,44 & K2IIII & $\mathrm{K} 2 \mathrm{IVa}$ & KIIII-IV \\
\hline 81797 & 46390 & 1,99 & 1,440 & 18,40 & 0,78 & 0,04 & 54 & $-1,75$ & K3IIIa & $\mathrm{K} 3 \mathrm{Ilb}$ & N3Il-IIl var. \\
\hline 98262 & 55219 & 3,49 & 1,400 & 7,74 & 0,79 & 0,10 & 129 & $-2,02$ & K3-III & K3II & \\
\hline 129078 & 72370 & 3,83 & 1,433 & 7,93 & 0,53 & 0,07 & 126 & $-1,82$ & K3HII CNO,5 & $\mathrm{K} 3 \mathrm{IIb}$ & \\
\hline 136422 & 75177 & 3,57 & 1,534 & 9,99 & 0,79 & 0,08 & 100 & $-1,55$ & KAIII & K4llb & KSSIII \\
\hline 151101 & 81660 & 4,84 & 1,212 & 4,79 & 0,45 & 0,09 & 209 & $-2,01$ & KOIII CN-0,5 CH-2 CaI & Koll & Klp \\
\hline 161239 & 86731 & 5,73 & 0,683 & 26,13 & 0,63 & 0,02 & 38 & 2,77 & $G 2111 b$ & $\mathrm{G} 2 \mathrm{IV}$ & \\
\hline 168454 & 89931 & 2,72 & 1,380 & 10,67 & 0,93 & 0,09 & 94 & $-2,25$ & $K 2,5111 a \mathrm{a} N \mathrm{~N} 0,5$ & K2,5II & \\
\hline 214850 & 111974 & 5,72 & 0,723 & 30,49 & 0,95 & 0,03 & 33 & 3,63 & G4V & G5IVb & \\
\hline 215665 & 112440 & 3,97 & 1,070 & 8,26 & 0,70 & 0,08 & 121 & $-1,59$ & G8111aCNO,5 & G8116 & G8lab: G8II-III \\
\hline 217014 & 113357 & 5,45 & 0,666 & 65,10 & 0,76 & 0,01 & 15 & 4,50 & G2IV & $\mathrm{G} 2: \mathrm{Va}:$ & G2,5IVa GAV GSV G01ar. Gislh \\
\hline 221148 & 115953 & 6,26 & 1,123 & 20,44 & 0,86 & 0,04 & 49 & 2,95 & $K 3-I I I b$ & K3IV: & K3HIVar. K2III-IV \\
\hline
\end{tabular}

\begin{tabular}{|c|c|c|c|c|c|c|}
\hline$\sigma(\pi) / \pi$ & $0-0,05$ & $0,06-0,10$ & $0,11-0,15$ & $0,16-0,25$ & $0,26-0,50$ & total \\
$n$ (étoiles) & 209 & 128 & 63 & 51 & 35 & 486 \\
\hline Désaccord & 6 & 8 & 8 & 13 & 19 & 54 \\
(1 cl. Lumi.) & $2,9 \%$ & $6,3 \%$ & $12,7 \%$ & $25,5 \%$ & $54,3 \%$ & $11,1 \%$ \\
\hline Désaccord & 17 & 29 & 22 & 11 & 9 & 88 \\
$(1 / 2$ cl. Lumi.) & $8,1 \%$ & $22,7 \%$ & $34,9 \%$ & $21,6 \%$ & $25,7 \%$ & $18,1 \%$ \\
\hline Désaccord & 23 & 37 & 30 & 24 & 28 & 142 \\
& $11,0 \%$ & $28,9 \%$ & $47,6 \%$ & $47,1 \%$ & $80,0 \%$ & $29,2 \%$ \\
\hline Accord & 186 & 91 & 33 & 27 & 7 & 344 \\
& $89,0 \%$ & $71,1 \%$ & $52,4 \%$ & $52,9 \%$ & $20,0 \%$ & $70,8 \%$ \\
\hline
\end{tabular}

- Nous avons considéré également 3 autres zones : 0,11 0,$15 ; 0,16-0,25 ; 0,26-0,50$; pour chacune de ces zones les désaccords d'une classe de luminosité sont respectivement de $12,7 \%, 25,5 \%$ et 54,3\%. L'ensemble de ces résultats est résumé dans le tableau ci-dessus.

Cette étude met donc en évidence l'impossibilité d'utiliser fiablement les calibrations de Schmidt-Kaler concernant les étoiles les plus lumineuses (supergéantes et même géantes brillantes) car toutes, pratiquement, appartiennent aux trois dernières zones pour lesquelles les erreurs relatives des parallaxes atteignent des valeurs élevées.

\subsection{Apport des données Hipparcos pour les étoiles à spectre composite}

Comme nous l'avons indiqué en introduction, nous comptions utiliser les magnitudes absolues déduites des parallaxes Hipparcos pour tester et préciser nos résultats quant aux classifications spectrales des composantes des binaires à spectre composite.
Malheureusement, il se trouve que ces objets sont presque tous relativement éloignés du Soleil, typiquement à quelques centaines de parsecs ; les erreurs relatives sur les parallaxes sont donc élevées : pour seulement 15 étoiles cette quantité est inférieure à $10 \%$, condition nécessaire, nous l'avons vu, pour assurer une certaine fiabilité des données.

En Tableau 2 nous donnons la liste de ces 15 binaires avec les indications suivantes :

- nos classifications Sp1(IR) dans le proche infrarouge des composantes froides (Ginestet et al. 1997, 1999) ;

- nos classifications Sp2 dans le bleu-proche UV des composantes chaudes (travail en cours) ou, à défaut, les meilleures classifications tirées de la littérature ;

- les indices de couleurs $(B-V)_{0}$ Hip. et les magnitudes absolues $M_{v}$ corr. obtenues à partir des données Hipparcos mais corrigés du rougissement (voir Sect. 2) : ces quantités sont confrontées à celles calculées avec les calibrations de Schmidt-Kaler ; un léger ajustement des magnitudes absolues des composantes froides a été effectué si nécessaire afin de "s'approcher" au mieux des indices $B-V$ observés. 
Tableau 2. Confrontation des données Hipparcos avec les classifications spectrales pour un échantillon d'étoiles binaires à spectre composite ayant $\sigma(\pi) / \pi \leq 0,10$. Colonnes 2, 3, 4, 5, 6 : données Hipparcos ; $M_{v}$ : magnitude absolue obtenue à partir des données Hipparcos ; $(B-V)_{0}$ Hip., $M_{v}$ corr. : données Hipparcos corrigées du rougissement (voir Sect. 2) ; Sp1, Sp2 : types spectraux des 2 composantes ; $M_{v 1}$, magnitude absolue de la primaire ; $(B-V)$ calculé, $M_{v}$ calculée : paramètres du système calculés à partir des types spectraux et des calibrations de Schmidt-Kaler

\begin{tabular}{|c|c|c|c|c|c|c|c|c|c|c|c|c|c|c|c|}
\hline HD & $N^{\circ}$ HIPP. & $V$ & $B-V$ & $\begin{array}{c}\pi \\
\operatorname{mas}\end{array}$ & $\begin{array}{l}\sigma(\pi) \\
\text { mas }\end{array}$ & $\sigma(\pi) / \pi$ & $\begin{array}{c}\text { distance } \\
p c \\
\end{array}$ & $M_{V}$ & $M_{V}$ corr & $(B-V)_{0} H i p$. & Spl (1R) & $M_{V 1}$ & Sp2 & $M_{V}$ calculé & $B-V$ calculé \\
\hline 17878 & 13531 & 3,93 & 0,758 & 13,15 & 0,85 & 0,06 & 76 & -0.48 & $-0,57$ & 0,73 & G7III+ & -0.3 & $A 3 \mathrm{~V}$ & $-0,49$ & 0,73 \\
\hline 18925 & 14328 & 2,91 & 0,716 & 12,72 & 0,71 & 0,06 & 79 & $-1,57$ & $-1,66$ & 0,68 & G9l/1+ & $-0,9$ & $A I V$ & $-1,07$ & 0,78 \\
\hline 20084 & 16489 & 5,62 & 0,894 & 9,18 & 0,55 & 0,06 & 109 & 0,43 & 0,30 & 0,85 & G8III & 0.4 & FOV & 0,28 & 0,85 \\
\hline 23838 & 17932 & 5,65 & 0,779 & 9,41 & 0,85 & 0,09 & 106 & 0,52 & 0,39 & 0,74 & GTIII & 0.8 & FOV & 0,63 & 0,79 \\
\hline 25007 & 19461 & 5,10 & 0,589 & 9,44 & 0,86 & 0,09 & 106 & $-0,03$ & $-0,15$ & 0,55 & G8III & 0,6 & $A 2 \mathrm{~V}$ & 0,14 & 0,55 \\
\hline 35162 & 25045 & 5,06 & 0,662 & 11,09 & 0,85 & 0,08 & 90 & 0,28 & 0,18 & 0,63 & G6III & 0,4 & $A 3 \mathrm{~V}$ & 0,06 & 0,61 \\
\hline 74874 & 43109 & 3,38 & 0,685 & 24,13 & 1,29 & 0,05 & 41 & 0,29 & 0,24 & 0,67 & $G 7 I I I$ & 0,8 & $A 6 V$ & 0,51 & 0,68 \\
\hline 84367 & 47758 & 4,78 & 0,516 & 8,49 & 0,73 & 0,09 & 118 & $-0,58$ & $-0,72$ & 0,47 & G7III & 0,5 & Am/delta Del. & $-0,25$ & 0,47 \\
\hline 102509 & 57565 & 4,50 & 0,547 & 14,40 & 0,86 & 0,06 & 69 & 0,29 & 0,21 & 0,52 & G5III- & 1,2 & $A 5 \mathrm{~V}$ & 0,74 & 0,56 \\
\hline 107700 & 60351 & 4,78 & 0,515 & 11,93 & 0,72 & 0,06 & 84 & 0,16 & 0,06 & 0,48 & G7III & 0,8 & $12 \mathrm{~V}$ & 0,27 & 0,49 \\
\hline 175492 & 92818 & 4,57 & 0,782 & 6,71 & 0,63 & 0,09 & 149 & $-1,30$ & $-1,48$ & 0,72 & GSIII+ & -1 & $A / \mathrm{K}$ & $-1,16$ & 0,77 \\
\hline 183912 & 95947 & 3,05 & 1,088 & 8,46 & 0,58 & 0,07 & 118 & $-2,31$ & $-2,45$ & 1,04 & $K 2 I I$ & $-2,3$ & $B 9,5 \mathrm{~V}$ & $-2,39$ & 1,10 \\
\hline 184759 & 96302 & 5,39 & 0,581 & 6,06 & 0,58 & 0,10 & 165 & $-0,70$ & $-0,90$ & 0,51 & G8III & 0,8 & $A 2 \mathrm{~V}$ & 0,27 & 0,51 \\
\hline 193495 & 100345 & 3,05 & 0,790 & 9,48 & 0,95 & 0.10 & 105 & $-2,07$ & $-2,19$ & 0,75 & G9II & -2.3 & $B 8 V$ & $-2,45$ & 0,80 \\
\hline 202447 & 104987 & 3,92 & 0,549 & 17,51 & 0,89 & 0,05 & 57 & 0,14 & 0,07 & 0,53 & G8III- & 1 & A3m & 0,47 & 0,55 \\
\hline
\end{tabular}

Nous constatons que l'accord entre les paramètres Hipparcos $M_{v}, B-V$ et ceux calculés à partir des calibrations de Schmidt-Kaler peut être considéré comme satisfaisant : seul le système HD 184759 ( $d=165$ pc, $\sigma(\pi) / \pi=0,10)$ montre un désaccord en $M_{v}$ supérieur à une magnitude.

\section{Conclusions}

Le comportement des 511 standards MK de types G, K, M vis-à-vis des données Hipparcos conduit aux constatations suivantes :

1. Pour de faibles valeurs des erreurs relatives sur les parallaxes $(\sigma(\pi) / \pi \leq 0,05)$, donc pour de faibles distances ne dépassant pas approximativement $75 \mathrm{pc}$, les données de SK et d'Hipparcos présentent un bon accord : les quelques étoiles discordantes devraient être reclassées dans le proche infrarouge. En effet, nos travaux (Ginestet et al. 1994; Carquillat et al. 1997) ont établi la validité de la région spectrale 8400 - $8800 \AA$ pour obtenir des classifications MK fiables ; de plus l'utilisation de ce domaine spectral constitue un avantage certain avec les récepteurs CCD très performants à ces longueurs d'onde ;

2. Les données avec $\sigma(\pi) / \pi>0,05$ devraient avoir une utilisation essentiellement statistique. Une vingtaine d'objets de classe III ayant des magnitudes absolues voisines de -2 devraient également être reclassées dans l'infrarouge. Pour l'instant la question des supergéantes reste encore en suspens car malheureusement ces étoiles sont presque toujours à des distances conséquentes et leurs parallaxes demeurent, même avec Hipparcos, trop incertaines ;

3. Cette dernière remarque s'applique aux étoiles à spectre composite dont la plupart sont des objets relativement lointains ;

4. Enfin, et surtout, les données fournies par Hipparcos, pour les étoiles froides naines et géantes, ne semblent pas remettre en cause les calibrations de Schmidt-Kaler. Même sans aucune limite de $\sigma(\pi) / \pi$ il n'y a pas de chevauchement en $M_{v}$ entre ces deux classes de luminosité ; de plus la classe IV paraît avoir son propre domaine, mais avec une certaine dispersion qui demanderait, là aussi, quelques contrôles de classifications dans le proche IR.

Remerciements. Les recherches bibliographiques ont été faites à l'aide de la base de données SIMBAD du CDS (Strasbourg, France). Le professeur C. Jaschek a collaboré à ce travail mais sa disparition brutale n'a pas permis sa participation à la rédaction finale.

\section{Bibliographie}

van Altena W.F., Lee J.T., Hoffleit E.D., 1995, General Catalogue of Trigonometric Stellar Parallaxes, 4th edition, Yale Univ. Obs.

Batten A.H., Fletcher J.M., MacCarthy D.G., 1989, Publ. Dom. Astrophys. Obs., Victoria, 17

Bond H.E., 1970a, ApJ 160, 1127

Bond H.E., 1970b, ApJS 22, 117

Carquillat J.M., Jaschek C., Jaschek M., Ginestet N., 1997, A\&AS 123,5

Carquillat J.M., Ginestet N., Jaschek C., 1997, The classification of binary stars with composite spectra: confrontation whith Hipparcos/Tycho data, in: Hipparcos-Venice '97, ESA SP-402, 465

Cayrel de Strobel G., Hauck B., François P., et al., 1992, A\&AS 95,273 
ESA, 1997, The Hipparcos and Tycho Catalogues, ESA SP1200

Garcia B., 1989, Bull. Inform. CDS 36, 27

Ginestet N., Carquillat J.M., Jaschek M., Jaschek C., 1994, A\&AS 108, 359

Ginestet N., Carquillat J.M., Jaschek C., Jaschek M., 1997, A\&AS 123, 135

Ginestet N., Carquillat J.M., Jaschek C., 1999, A\&AS 134, 473

Girardi L., Groenewegen M.A.T., Weiss A., Salaris M., 1998, MNRAS 301, 149

Gómez A.E., Luri X., Mennessier M.O., Torra J., Figueras F., 1997, The luminosity calibration of the HR diagram revisited by Hipparcos, in: Hipparcos - Venice '97, ESA SP- 402, 207
Hoffleit D., Jaschek C., 1982, The Bright Star Catalogue, Yale Univ. Obs.

Jaschek C., Gómez A.E., 1998, A\&AS 330, 619

Keenan P.C., McNeil R.C., 1976, An Atlas of Spectra of the Cooler Stars: Types G, K, M, S and C, Perkins Obs., Ohio State and Ohio Wesleyan Univ.

Paunzen E., 1999, A\&A 341, 784

Schmidt-Kaler Th., 1982, in: Landolt-Börnstein, New Series, Gr. 6, Vol. 2-B. Springer-Verlag, Berlin, Heidelberg, New-York, p. 1

Turon C., 1998, C. R. Acad. Sci. Paris, t. 326, Ser. II b, 775 